\title{
13. APPENDICES
}

\begin{tabular}{|c|c|}
\hline \multicolumn{2}{|l|}{ APPENDIX 1} \\
\hline \multicolumn{2}{|c|}{ DESCRIPTION OF SELECTED BIRTH DEFECTS } \\
\hline \multicolumn{2}{|c|}{ The following include descriptions of some of the birth defects included in this report : } \\
\hline Anencephaly & Absence of the cranial vault, with the brain tissue completely missing or markedly reduced. \\
\hline Spina bifida & Defective closure of the bony encasement of the spinal cord, through which the spinal cord may protrude. \\
\hline Encephalocele & Protrusion of brain through a congenital opening in the skull \\
\hline Hydrocephalus & Dilatation of the cerebral ventricles accompanied by an accumulation of cerebral fluid within the skull. \\
\hline Buphthalmos & Enlargement and distension of the fi brous coats of the eye. \\
\hline Hypospadias & The opening of the urethra lies on the underside of the penis or on the perineum. \\
\hline Epispadias & $\begin{array}{l}\text { Absence of the upper wall of the urethra. The opening of the urethra lies on the dorsum of the penis in males, } \\
\text { and anterior to or onto the clitoris in females. }\end{array}$ \\
\hline Chordee & Downward bowing of the penis. \\
\hline Talipes equinovarus & A deformity of the foot in which the heel is elevated and turned outward. \\
\hline Polydactyly & Presence of additional fingers or toes on hands or feet. \\
\hline Syndactyly & Attachment of adjacent fingers or toes on hands or feet. \\
\hline Craniosynostosis & Premature closure of the sutures of the skull. \\
\hline Exomphalos & Herniation of the abdominal contents into the umbilical cord. \\
\hline Gastroschisis & A defect in the abdominal wall not involving the umbilicus and through which the abdominal contents herniate. \\
\hline Cystic hygroma & A sac, cyst or bursa distended with fluid. \\
\hline
\end{tabular}

\section{APPENDIX 2}

\section{BIRTH DEFECT EXCLUSION LIST}

The following is a general list of minor defects and non-structural disorders which are excluded from the NSW Birth Defects Register:

Abnormal palmar creases

Accessory nipples

Balanced chromosomal translocation (unless occurring with structural defects)

Birthmarks (single, $<4$ cms diameter)

Bronchopulmonary dysplasia

Cerebral palsy

Clicky hips

Congenital infections (unless occurring with structural defects)

Congenital neoplasms/tumours (exception: cystic hygroma)

Developmental disability

Deviated nasal septum

Fetal alcohol syndrome

Glucose-6-phosphate dehydrogenase (G6PD) deficiency

Haemophilia

Heart murmurs (functional)

Hernia (epigastric, hiatus, inguinal, umbilical)

Hydrocele (testis)

Hypoplastic lung (less than 37 weeks gestation)

Imperforate hymen
Inborn errors of metabolism other than phenylketonuria and congenital hypothyroidism.

Intrauterine growth retardation

Low birthweight

Meconium ileus

Minor ear anomalies

Minor finger/hand anomalies

Minor toe/foot anomalies

Muscular dystrophies \& myopathies

Oesophageal reflux

Patent ductus arteriosus (less than 37 weeks gestation)

Pilonidal sinus

Sacral dimples

Single umbilical artery (unless occurring with structural defects)

Skin tag

Strabismus

Talipes (exception: those requiring surgery)

Tongue tie

Undescended testes (exception: those requiring surgery)

Webbing of 2 nd and 3rd toes

Wide sutures 


\section{APPENDIX 3}

MATERNAL COUNTRIES OF BIRTH AND COUNTRY OF BIRTH GROUPS

\begin{tabular}{|c|c|c|c|}
\hline English speaking & Eastern Europe, Russia, & Saudi Arabia & South East Asia \\
\hline Australia & Central Asian and Baltic & Syria & Brunei \\
\hline Christmas Island & States & Turkey & Cambodia \\
\hline Cocos (Keeling) Islands & Bulgaria & United Arab Emirates & Indonesia \\
\hline Norfolk Island & Czechoslovakia & West Bank & Laos \\
\hline New Zealand & Hungary & Yemen & Malaysia \\
\hline United Kingdon & Poland & Algeria & Burma (Myanmar) \\
\hline Channel Islands & Romania & Egypt & Philippines \\
\hline Isle of Man & Armenia & Libya & Singapore \\
\hline Ireland & Azerbaijan & Mauritania & Thailand \\
\hline Bermuda & Belarus (formerly & Morocco & Vietnam \\
\hline Canada & Byelorussia) & Sudan & \\
\hline United States of America & Estonia & Tunisia & Southern Asia \\
\hline \multirow[t]{2}{*}{ South Africa } & Georgia & Cameroon & Afganistan \\
\hline & Kazakhstan & Central African Republic & Bangladesh \\
\hline Central and South America & Kyrgyzstan (formerly & Congo & Bhutan \\
\hline Bahrain & Kirghizia) & Cote d'Ivoire & India \\
\hline Argentina & Latvia & Gambia & Maldives \\
\hline Bolivia & Lithuania & Ghana & Nepal \\
\hline Brazil & Moldova (formerly Moldavia) & Guinea-Bissau & Pakistan \\
\hline Chile & Russian Federation & Liberia & Sri Lanka \\
\hline Colombia & Ukraine & Mali & \\
\hline Ecuador & Uzbekistan & Nigeria & Southern Europe \\
\hline Falkland Islands & Kazakhstan & Senegal & Albania \\
\hline French Guiana & Kyrgystan & Sierra Leone & Andorra \\
\hline Guyana & & Zaire & Andorra \\
\hline Paraguay & Melanesia, Micronesia and & Angola & Cyprus \\
\hline Peru & Polynesia & Botswana & Gibraltar \\
\hline Surinam & New Caledonia & Djibouti & Greece \\
\hline Uruguay & Papua New Guinea & Ethiopia & Italy \\
\hline Venezuela & Solomon Islands & Kenya & Malta \\
\hline Belize & Vanuatu & Malawi & Portugal \\
\hline Costa Rica & Guam & Mauritius & Spain \\
\hline El Salvador & Kiribati & Mozambique & Bosnia-Herzegovina \\
\hline Guatamala & $\begin{array}{l}\text { Kiribatı } \\
\text { Nauru }\end{array}$ & $\begin{array}{l}\text { Mozambique } \\
\text { Namibia }\end{array}$ & Croatia \\
\hline Honduras & $\begin{array}{l}\text { Nauru } \\
\text { Cook Islands }\end{array}$ & & Macedonia \\
\hline $\begin{array}{l}\text { Honduras } \\
\text { Mexico }\end{array}$ & & Reunion & Slovenia \\
\hline $\begin{array}{l}\text { Mexico } \\
\text { Nicaragua }\end{array}$ & Fiji & Rwanda & Serbia and Montenegro \\
\hline & French Polynesia (including & Seychelles & Former Yugoslavia (not \\
\hline Panama & Tahiti) & Somalia & otherwise defined) \\
\hline Antigua and Barbuda & Niue & Swaziland & \\
\hline Bahamas & American Samoa & Tanzania & \\
\hline Barbados & Western Samoa & Uganda & Western and Northern \\
\hline Cayman Islands & Tokelau & Zambia & Europe \\
\hline Cuba & Tonga & Zimbabwe & Austria \\
\hline Grenada & Tuvalu & Eritrea & Belgium \\
\hline Guadeloupe & Wallis and Fortuna & Ethiopia & France \\
\hline Jamaica & & & Germany (United) \\
\hline Netherlands Antilles & & Nonth Kact Acia & Luxembourg \\
\hline Puerto Rico & Middle East and Africa & vhin Easta & Netherlands \\
\hline St Kitts-Nevis & Bahrain & china (exciuding raiwan) & Switzerland \\
\hline St Lucia & Gaza Strip & Hong Kong & Denmark \\
\hline St Vincent and the & Iran & Japan & Faeroe Islands \\
\hline & Iraq & North Korea & Finland \\
\hline Grenadines & Israel & South Korea & Iceland \\
\hline Trinidad and Tobago & Jordan & Macau & Norwav \\
\hline Turks and Caicos Islands & Kuwait & Mongolia & civeden \\
\hline & Lebanon & Taiwan & sweden \\
\hline & Qatar & & \\
\hline
\end{tabular}




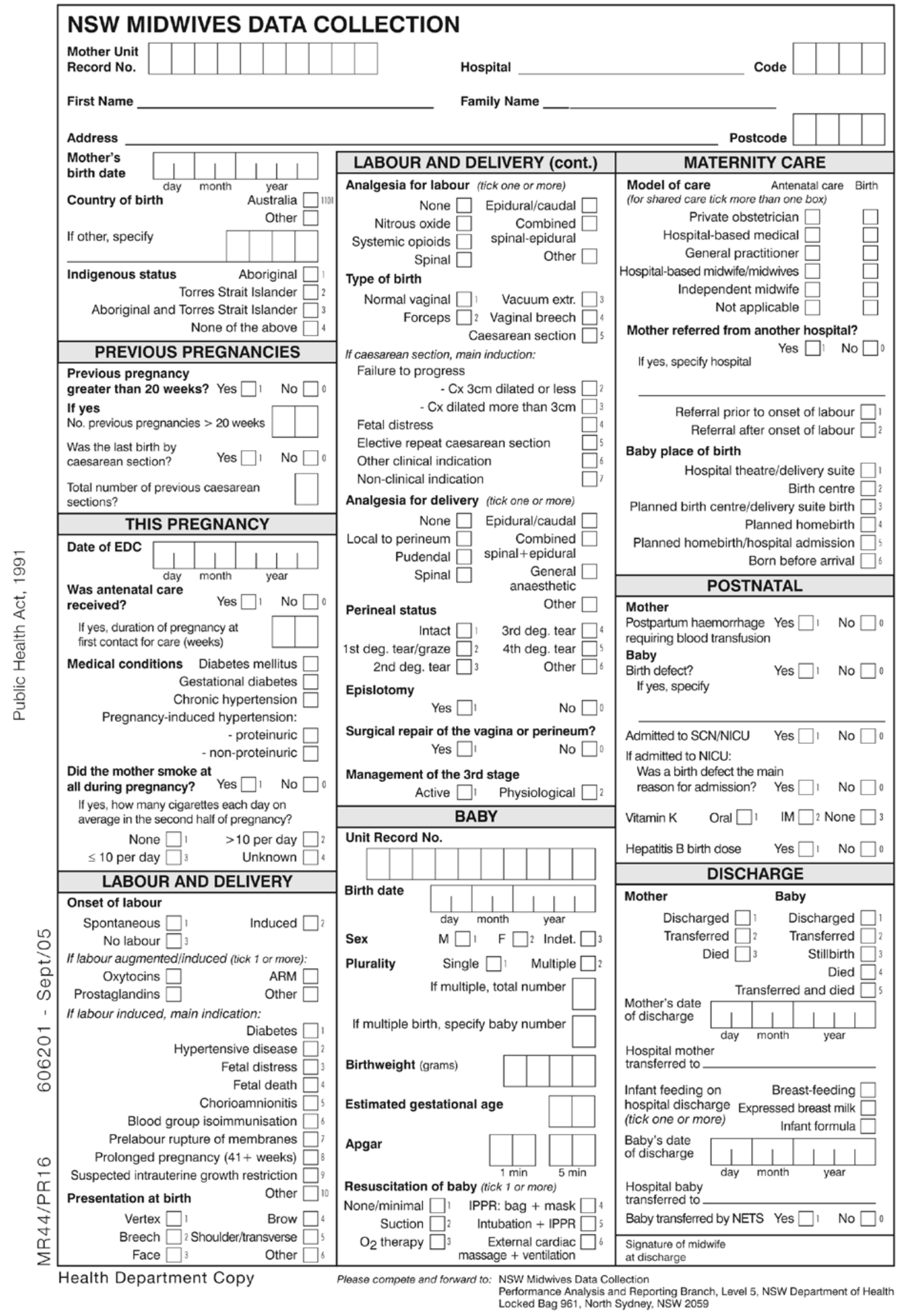

